\title{
The typical structure of graphs without given excluded subgraphs
}

\author{
József Balogh,* \\ Béla Bollobás ${ }^{\dagger}$ \\ and Miklós Simonovits
}

September 4, 2008

\begin{abstract}
Let $\mathcal{L}$ be a finite family of graphs. We describe the typical structure of $\mathcal{L}$-free graphs, improving our earlier results [2] on the ErdősFrankl-Rödl theorem [6], by proving our earlier conjecture that, for $p=p(\mathcal{L})=\min _{L \in \mathcal{L}} \chi(L)-1$, the structure of almost all $\mathcal{L}$-free graphs is very similar to that of a random subgraph of the Turán graph $T_{n, p}$. The "similarity" is measured in terms of graph theoretical parameters of $\mathcal{L}$.
\end{abstract}

\section{Introduction}

Notation. We restrict our attention to simple graphs and the notation we use is standard. Thus $V(G)$ denotes the set of vertices of a graph $G$, and for a vertex set

\footnotetext{
*University of Illinois at Urbana-Champaign, Urbana, IL 61801, USA; email: jobal@math.uiuc.edu, research supported in part by NSF grants DMS-0302804, DMS0603769 and DMS-0600303, UIUC Campus Research Board 06139 and 07048, and OTKA 049398.

${ }^{\dagger}$ Trinity College, Cambridge CB2 1TQ, UK and Department of Mathematical Sciences, University of Memphis, Memphis TN 38152; email: B.Bollobas@dpmms.cam.ac.uk, partially supported by NSF ITR grants CCR-0225610, DMS-0505550, and ARO grant W911NF-06-1-0076.

${ }^{\ddagger}$ Rényi Institute, Budapest, Hungary; email: miki@renyi.hu, partially supported by OTKA grants K-69062, NK 62321.
} 
$X \subseteq V(G), G[X]$ denotes the subgraph of $G$ induced by $X$. For $X \subseteq V(G)$, we mostly shorten $e(G[X])$ to $e(X)$. We write $G_{n}$ for a graph of order $n$; in fact, much of the time, the first suffix in our notation is the order of the graph, as in $K_{p}, T_{n, p}$ and $H_{k}$. The chromatic number of a graph $L$ is denoted by $\chi(L)$, the order of $L$ by $v(L) ; \Gamma(x)$ is the set of neighbours of a vertex $x, d(x)=|\Gamma(x)|$ is its degree, and $d(x, A)=|\Gamma(x) \cap A|$ is the degree of $x$ into a set $A \subseteq V(G)$. Also, $\Gamma^{*}(X)$ denotes the set of common neighbours of the vertices in $X: \Gamma^{*}(X)=\bigcap_{x \in X} \Gamma(x)$.

We write $K_{p}$ for the complete graph on $p$ vertices, and $T_{n, p}$ for the $p$-class Turán graph: Thus to obtain $T_{n, p}$ we partition $n$ vertices into $p$ classes so that their sizes are as equal as possible, and join two vertices if they belong to different classes. It is easy to see that

$$
\left(1-\frac{1}{p}\right)\left(\begin{array}{l}
n \\
2
\end{array}\right) \leq e\left(T_{n, p}\right) \leq\left(1-\frac{1}{p}\right) \frac{n^{2}}{2} \quad \text { and } \quad e\left(T_{n, p}\right)=\left(1-\frac{1}{p}\right) \frac{n^{2}}{2}+O(n) .
$$

For a given graph $G_{n}$ and $p$, a $p$-partition is a partition of $V\left(G_{n}\right)$ into $p$ classes, a $p$-partition $\left(U_{1}, \ldots, U_{p}\right)$ of $V\left(G_{n}\right)$ is optimal if $\sum e\left(U_{i}\right)$ is as small as possible. Sometimes, shortly we refer to such a partition as an optimal p-partition.

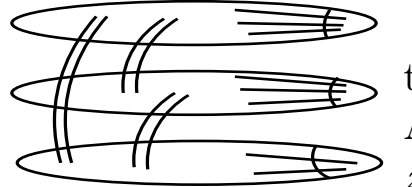

Given a partition $\left(U_{1}, \ldots, U_{p}\right)$ of $V\left(G_{n}\right)$, we shall call the edges inside some partition-class $U_{i}$ "horizontal edges" 1 Also, for a given partition $\left(U_{1}, \ldots, U_{p}\right)$ we define the horizontal degree of $x \in U_{i}$ to be $\left|\Gamma(x) \cap U_{i}\right|$.

We say that a pair of vertex sets $(A, B)$ is completely joined in a graph $G_{n}$ if $A, B \subset V\left(G_{n}\right), A \cap B=\emptyset$, and each $x \in A$ is joined to each $y \in B$ in $G_{n}$. Having two vertex-disjoint graphs $M$ and $Q, M \otimes Q$ denotes the graph obtained by joining each vertex of $M$ to each vertex of $Q$.

In this paper the logarithms have always base 2 . We shall often use the binary entropy function $H(x)=x \log _{2} \frac{1}{x}+(1-x) \log _{2} \frac{1}{1-x}$.

\subsection{Turán type extremal problems}

We say that the graph $G$ contains $L$ and write $L \subseteq G$ if $L$ is a (not necessarily induced) subgraph of $G$. Given a family $\mathcal{L}$ of graphs, $G$ is called $\mathcal{L}$-free if $G$ contains no $L \in \mathcal{L}$, We call $\mathcal{L}$ the family of forbidden graphs. We assume that $e(L)>0$ for each $L \in \mathcal{L} . \mathcal{P}(n, \mathcal{L})$ denotes the class of $\mathcal{L}$-free graphs with vertex set

\footnotetext{
${ }^{1}$ Mostly we call these as "horizontal degrees" that corresponds to specific figures of the optimal partition where these edges are almost horizontal.
} 
$[n]:=\{1, \ldots, n\} ; 2 \operatorname{ex}(n, \mathcal{L})$ is the maximum number of edges an $\mathcal{L}$-free graph $G_{n}$ can have, and an $\mathcal{L}$-free graph with $\operatorname{ex}(n, \mathcal{L})$ edges is $\mathcal{L}$-extremal or sometimes simply extremal. When $\mathcal{L}$ consists of a single graph $L$, we write $\operatorname{ex}(n, L)$ instead of $\operatorname{ex}(n,\{L\})$.

The basic Turán type extremal problem is as follows.

For a given family $\mathcal{L}$, determine or estimate $\operatorname{ex}(n, \mathcal{L})$, and describe the (asymptotic) structure of extremal graphs, as $n \rightarrow \infty$.

We fix a forbidden family $\mathcal{L}$, and let

$$
p:=p(\mathcal{L})=\min _{L \in \mathcal{L}} \chi(L)-1 .
$$

For every $\mathcal{L}$ there is a constant $a>0$ such that

$$
\operatorname{ex}(n, \mathcal{L})=e\left(T_{n, p}\right)+O\left(n^{2-a}\right)
$$

and all the extremal graphs of order $n$ can be transformed into $T_{n, p}$ by deleting and adding $O\left(n^{2-a}\right)$ edges, as proved by Erdös [5] and Simonovits [8]. For a more detailed description of this field, see the book of Bollobás [3] or the surveys of Simonovits [9], 10], Füredi [7] and Bollobás [4].

The main idea of the results discussed here and in the preceding papers is that most of the $\mathcal{L}$-free graphs can be regarded as subgraphs of some extremal or almost extremal graphs for $\mathcal{L}$. Our starting point was the following theorem of Erdős, Frankl and Rödl [6].

Theorem 1. For every $\mathcal{L}$

$$
2^{\operatorname{ex}(n, \mathcal{L})} \leq|\mathcal{P}(n, \mathcal{L})| \leq 2^{\operatorname{ex}(n, \mathcal{L})+o\left(n^{2}\right)} .
$$

Note that the lower bound in (3) is trivial, as every subgraph of an $\mathcal{L}$-extremal graph is $\mathcal{L}$-free. In [2] we improved the upper bound in Theorem 1, see Theorem 3 , Here we go one step further, and give a structural characterization of almost all graphs in $\mathcal{P}(n, \mathcal{L})$. To formulate our results, we need a definition.

Definition 2 (Decomposition Family). Given a family $\mathcal{L}$ (and $p=p(\mathcal{L})$ ), let $\mathcal{M}:=\mathcal{M}(\mathcal{L})$ be the family of minimal graphs $M$ for which there exist an $L \in \mathcal{L}$ and a $t=t_{L}$ such that $L \subseteq M^{\prime} \otimes K_{p-1}(t, \ldots, t)$, where $M^{\prime}$ is the graph obtained by adding $t$ isolated vertices to $M$. We call $\mathcal{M}$ the decomposition family of $\mathcal{L}$.

\footnotetext{
${ }^{2}$ The vertices of our graphs are fixed, labelled and, for the sake of simplicity, we shall assume that $V\left(G_{n}\right)=\{1, \ldots, n\}$.
} 
In other words, a graph $M$ belongs to $\mathcal{M}$ if whenever $n$ is sufficiently large and we "place" $M$ into a class $U_{i}$ of $T_{n, p}$, then the obtained graph contains a forbidden $L \in \mathcal{L}$. ("Placing" means adding the edges of a copy of $M$ into $T_{n, p}$, using only vertices of this $U_{i}$.) We emphasize that $\mathcal{M}$ always contains a bipartite graph, otherwise $\chi(L) \geq p+2$ for every $L \in \mathcal{L}$.

If $\mathcal{L}$ is finite, then $\mathcal{M}$ is also finite. The converse is not necessarily true. For example, if $\mathcal{L}$ is the family of all the odd cycles, then $\mathcal{M}=\left\{K_{2}\right\}$.

In [2] we gave the following improvement of Theorem 1],

Theorem 3. For every $\mathcal{L}$ with $p=p(\mathcal{L}) \geq 2$, if $\mathcal{M}$, the decomposition family of $\mathcal{L}$, is finite, then

$$
|\mathcal{P}(n, \mathcal{L})| \leq n^{\mathbf{e x}(n, \mathcal{M})+c_{\mathcal{L}} \cdot n} \cdot 2^{\frac{1}{2}\left(1-\frac{1}{p}\right) n^{2}}
$$

for a sufficiently large constant $c_{\mathcal{L}}>0$.

To see that this does strengthen Theorem 11, for a given $\mathcal{L}$, let $L \in \mathcal{L}$ have minimum chromatic number, (i.e. $\chi(L)=p+1$ ), and pick a $t$ with $K_{p+1}(t, t, \ldots, t)$ $\supseteq L$. This implies that there is an $M \in \mathcal{M}$ with $M \subset K(t, t)$ and Theorem 3 implies that

$$
|\mathcal{P}(n, \mathcal{L})| \leq|\mathcal{P}(n, L)| \leq n^{\mathbf{e x}(n, M)+c_{L} \cdot n} \cdot 2^{\frac{1}{2}\left(1-\frac{1}{p}\right) n^{2}} \leq 2^{\operatorname{ex}(n, \mathcal{L})+O\left(n^{2-c}\right)}
$$

where $0<c<1 / t$. Here we used (2), $\operatorname{ex}(n, K(t, t))=O\left(n^{2-1 / t}\right)$ and $n^{O\left(n^{2-1 / t}\right)}<$ $2^{O\left(n^{2-c}\right)}$.

Remark 4. One knowing this field may ask: do we need/use the Szemerédi Regularity Lemma [1] in this paper or not? The answer is that in some crucial steps of our previous paper [2] we did use and here we use several results from that paper. However, here we do not need the explicit use of the Regularity Lemma.

\subsection{Why do we need the finiteness of $\mathcal{M}$ ?}

We construct a family $\mathcal{L}$ which shows that the condition of finiteness of $\mathcal{M}$ is needed in Theorem 3. Denote by $I_{\nu}$ the $\nu$-vertex graph with no edges. Set $f(x)=2^{x^{2}}$ and let $L_{m}:=C_{m} \otimes I_{f(m)}$, i.e. join $f(m)$ independent vertices to an $m$-cycle $C_{m}$ completely. Put

$$
\mathcal{L}:=\left\{C_{m} \otimes I_{f(m)}: m=3,4,5, \ldots\right\} .
$$

We assert that for this $\mathcal{L}$ the conclusions of Theorem 3 (and Theorem 5 below) do not hold: for infinite $\mathcal{L}$ they are not necessarily true. 
(a) Now, $\mathcal{M}$ is the family of all cycles, $p=p(\mathcal{L})=2$ and $\operatorname{ex}(n, \mathcal{M})=n-1$. Hence (4) would yield that

$$
|\mathcal{P}(n, \mathcal{L})| \leq n^{\left(c_{\mathcal{L}}+1\right) \cdot n} \cdot 2^{\frac{1}{2}\left(1-\frac{1}{p}\right) n^{2}} \leq 2^{n^{2} / 4+O(n \log n)} .
$$

(b) On the other hand, let $G_{m}$ be a graph on $m=\lceil n / 2\rceil$ vertices with average degree at least $\log ^{2} n$, and girth $g\left(G_{m}\right) \rightarrow \infty$. (Random graph methods or the Margulis-Lubotzky-Phillips-Sarnak type graphs imply that there are such graphs with girth $g\left(G_{m}\right)>c_{1} \frac{\log m}{\log \log m}$.) Let $S_{n}:=G_{m} \otimes I_{n-m}$. With our condition on $f(x)$ we have $f\left(g\left(G_{m}\right)\right)>2 m$. One can easily see that $S_{n}$ is $\mathcal{L}$-free and

$$
|\mathcal{P}(n, \mathcal{L})| \geq 2^{e\left(S_{n}\right)}>2^{e\left(T_{n, 2}\right)} \cdot 2^{c n \log ^{2} n},
$$

contradicting (5). So Theorem 3 does not hold for this infinite $\mathcal{L}$.

\subsection{Results}

The goal of this paper is to prove Conjecture 2.3 from [2]. We actually prove a stronger result, Theorem 9, which provides an estimate for the decay of the number of "bad graphs" compared to the number of $\mathcal{L}$-free graphs (and gives additional structural information on almost all $\mathcal{L}$-free graphs).

Theorem 5. Let $\mathcal{L}$ be a finite family of graphs. Then there exists a constant $h_{\mathcal{L}}$ such that for almost all $\mathcal{L}$-free graphs $G_{n}$ we can delete $h_{\mathcal{L}}$ vertices of $G_{n}$ and partition the remaining vertices into $p$ classes $\left(U_{1}, \ldots, U_{p}\right)$ so that $G\left[U_{i}\right]$ is an $\mathcal{M}$-free graph for every $1 \leq i \leq p$.

Observe that the family $\mathcal{L}$ constructed in Section 1.2 shows that in Theorem 5 at least the condition that $\mathcal{M}$ is finite is needed.

We shall define several classes of $\mathcal{L}$-free graphs; each one will be used to describe the similarity of typical $\mathcal{L}$-free graphs to random subgraphs of some $\mathcal{L}$-extremal graphs.

Definition 6 (Fixing the parameters I). Let

$$
t:=\max _{L \in \mathcal{L}} v(L) .
$$

As in (11), let $p+1$ be the minimum chromatic number of a member of $\mathcal{L}$ and let $\delta<n /\left(p 4^{t}\right)$ be a positive constant. We fix the constants

$$
\beta_{r}:=\frac{1}{2^{2 r+1}}
$$


and an $\varepsilon>0$ and a $\gamma>0$ satisfying that

$$
H(\varepsilon)<\frac{\beta_{t}}{100 p^{4} t^{2}} \quad \text { and } \quad \gamma \leq \frac{\beta_{t}}{100 p^{5} t^{5}}
$$

Here, we made use of the fact that $\mathcal{L}$ is finite: for infinite $\mathcal{L}$ this definition gives $\varepsilon=0$.

Definition 7 (Good $r$-tuples). Given a graph $G$ with a vertex partition $\left(U_{1}, \ldots, U_{p}\right)$, call an $r$-tuple $X \subset V(G)$ GOOD if for every $U_{i}$ disjoint from $X$ the number of common neighbours of the vertices of $X$ in $U_{i}$ is

$$
\left|\Gamma^{*}(X) \cap U_{i}\right|=\left|\bigcap_{x \in X} \Gamma(x) \cap U_{i}\right|>\frac{1}{4^{r+1}}\left|U_{i}\right| .
$$

An $r$-tuple is BAD if it is not GOOD. We say that $X$ is a BAD $r$-tuple for a class $U_{i(X)}$, if (9) is violated. Note that a set $X$ may be BAD for several classes in a partition, and whether $X$ is GOOD or BAD depends on $\left(U_{1}, \ldots, U_{p}\right)$.

Definition 8 (GOOD graphs). Denote by $\mathcal{P}_{\text {GOOD }}^{h}(n, \mathcal{L})$ the family of $\mathcal{L}$-free graphs $G_{n}$ having an optimal partition $\left(U_{1}, \ldots, U_{p}\right)$ in which, if $W$ is the set of vertices having horizontal degree at least $\varepsilon n$, (where we use the $\varepsilon$ fixed in Definition 6) then $|W| \leq h$ and the vertex set $V(G)-W$ contains no BAD $r$-tuples for $1 \leq r \leq t$ in $G_{n}$.

Theorem 9. There is an $h=h(\mathcal{L})$ such that almost all $\mathcal{L}$-free graphs are in $\mathcal{P}_{\text {GOOD }}^{h}(n, \mathcal{L})$ : there exist two positive constants, $C$ and $\omega>1$, such that

$$
\left|\mathcal{P}(n, \mathcal{L})-\mathcal{P}_{\text {GOOD }}^{h}(n, \mathcal{L})\right| \leq \frac{C}{\omega^{n}}|\mathcal{P}(n, \mathcal{L})|
$$

The constants $C$ and $\omega>1$ in Theorem 9] can be computed from its proof (which is unlikely to provide the best possible values). In a forthcoming paper we plan to discuss some consequences of Theorems [5] and 9. In the proof of Theorem 9] we shall use some lemmas of [2].

\subsection{Classes of $\mathcal{L}$-free graphs}

We shall use several subclasses of $\mathcal{P}(n, \mathcal{L})$. Often we shall neglect indicating the dependence on all the parameters. We shall define $\vartheta$ later, in Definition 15, and then fix $\delta:=2 \sqrt{H(\vartheta)}$. 
1. Let $\mathcal{P}_{\vartheta}(n, \mathcal{L})$ be the family of $\mathcal{L}$-free graphs on $[n]$ having (optimal) partitions $\left(U_{1}, \ldots, U_{p}\right)$ for which $\sum_{i} e\left(U_{i}\right)<\vartheta n^{2}$. These are the $\vartheta$ Turán graphs.

2. Let $3 \mathcal{P}_{\text {UNIF }}^{\delta}(n, \mathcal{L}) \subset \mathcal{P}_{\vartheta}(n, \mathcal{L})$ be the family of graphs for which every optimal p-partition is such that for every $1 \leq i<j \leq p$ and every pair of sets $A \subset U_{i}, \quad B \subset U_{j}$ with $|A|=|B| \geq\lceil\delta n\rceil$ the inequality $e(A, B)>(1 / 4)|A| \cdot|B|$ holds. We shall call these graphs $\delta$-lower regular (where "lower" refers to the fact that we have a lower bound on the density).

3. As in [2], we denote by $\mathcal{P}_{\text {WP }}^{\vartheta}(n, \mathcal{L})$ the family of graphs $G_{n} \in \mathcal{P}_{\vartheta}(n, \mathcal{L})$ all optimal partitions $\left(U_{1}, \ldots, U_{p}\right)$ of which satisfy

$$
|| U_{i}\left|-\frac{n}{p}\right|<\left(\sqrt{\vartheta} \log \frac{1}{\vartheta}\right) n
$$

for all $i$. (WP stands for "well partitioned".)

Let us fix a constant $\vartheta$ with $0<\vartheta<(3 p)^{-12}$. (Later we shall have some further restrictions on $\vartheta$.) The "Main Lemma" of [2] asserts that almost all $\mathcal{L}$-free graphs are $\vartheta$-Turán graphs. Note that here we quote the results that we actually proved in [2], not the weaker form as we stated them there 4] Unfortunately, in [2] we often replaced $2^{-\rho n^{2}}$ by the rather weak bound $2^{-n}$.

Lemma 10. (Main Lemma in [2]) Let $0<\vartheta<(3 p)^{-12}$. Then, for a suitable positive constant $\rho=\rho(\vartheta)>0$ and an integer $n_{0}(\vartheta)$, for $n>n_{0}(\vartheta)$ we have

$$
\left|\mathcal{P}(n, \mathcal{L})-\mathcal{P}_{\vartheta}(n, \mathcal{L})\right| \leq 2^{e\left(T_{n, p}\right)-\rho n^{2}} .
$$

Lemma 11. (Lemma 6.1 in [2]) Let $0<\vartheta<(3 p)^{-12}$. Then for $\delta \geq 2 H(\vartheta)$ there is a positive constant $\rho=\rho(\vartheta, \delta)$ such that for $n$ sufficiently large we have

$$
\left|\mathcal{P}(n, \mathcal{L})-\mathcal{P}_{\mathbf{U N I F}}^{\delta}(n, \mathcal{L})\right|<2^{e\left(T_{n, p}\right)-\rho n^{2}} .
$$

\footnotetext{
${ }^{3}$ As $\delta$ is a function of $\vartheta$, here we neglect to show in the notation the dependency of the family on $\vartheta$.

${ }^{4}$ The weaker bounds would be sufficient as well for our purposes, but now we think that stating the sharp results is better from point of view of understanding the proof better.
} 
Lemma 12. (Lemma 6.6 in [2]) Let $0<\vartheta<(3 p)^{-12}$. Then, for a suitable positive constant $\rho=\rho(\vartheta)>0$ and for $n$ sufficiently large we have

$$
\left|\mathcal{P}_{\vartheta}(n, \mathcal{L})-\mathcal{P}_{\mathbf{W P}}^{\vartheta}(n, \mathcal{L})\right|<2^{e\left(T_{n, p}\right)-\rho n^{2}} .
$$

We shall say that a family of graphs is negligible if its cardinality is at most $2^{e\left(T_{n, p}\right)-\rho n^{2}}$ for some constant $\rho>0$.

Remark 13. Lemmas 11 and 12 assert that the typical vertex-distribution and edge-distribution are very even in our optimal partitions.

Lemma 14. (Lemma 7.1 in [2]) Given $\mathcal{L}$, let $p$ be defined by [1). For any $\varepsilon>0$ there is a $0<\delta(\varepsilon)<1 / p$ such that if $\vartheta>0$ satisfies that $\delta:=2 \sqrt{H(\vartheta)}<\delta(\varepsilon)$, then the following holds: there exist two integers $h_{0}(\vartheta, \varepsilon, \mathcal{L})$ and $n_{0}(\vartheta, \varepsilon, \mathcal{L})$ for which, if $G_{n} \in \mathcal{P}_{\mathbf{U N I F}}^{\delta}(n, \mathcal{L})$ and $n>n_{0}$, and if $V\left(G_{n}\right)=\left(U_{1}, \ldots, U_{p}\right)$ is an optimal partition of $G_{n}$, then for every $1 \leq i \leq p$

$$
\left|\left\{x \in U_{i}: d\left(x, U_{i}\right) \geq \varepsilon n\right\}\right| \leq h_{0}(\vartheta, \varepsilon, \mathcal{L}) .
$$

Roughly speaking, Lemma 14 states that in an optimal partition "the number of vertices with 'high' horizontal degree is bounded".

Definition 15 (Fixing the parameters II). In Definition 6 we already determined (for a given $\mathcal{L}$ ) the integers $p$ and $t$ and the constants $\beta_{r}, \gamma$ and $\varepsilon$. For this $\varepsilon$, we choose a $\delta(\varepsilon)$ as in Lemma 14, and our $\delta>0$ and $\vartheta$ satisfying $\delta=2 \sqrt{H(\vartheta)}<\delta(\varepsilon)$. Let $\rho>0$ be defined to be the minimum of the $\rho^{\prime} s$ provided by Lemmas [10, 11] and 12. Make sure that $\vartheta, \delta$ and $\rho$ are small enough (compared to $\gamma$ ) to satisfy

$$
2 H(\vartheta)+\rho<\frac{\beta_{t} \gamma}{4 p t}
$$

All these constants should be (and can be) chosen small enough to satisfy

$$
2 p H(p \delta+2 p \gamma)+H(\varepsilon)+4 \sqrt{\vartheta} \log \frac{1}{\vartheta}+\gamma<\frac{\beta_{t}}{10 p t} .
$$

Finally, we fix $h:=p \cdot h_{0}(\vartheta, \varepsilon, \mathcal{L})$, where $h_{0}$ is the constant whose existence is provided by Lemma 14, and let $\ell:=\lceil\gamma n\rceil$.

Let

$$
\mathcal{Q}_{\text {GOOD }}^{h}(n, \mathcal{L}):=\mathcal{P}_{\text {GOOD }}^{h}(n, \mathcal{L}) \cap \mathcal{P}_{\text {WP }}^{\vartheta}(n, \mathcal{L}) \cap \mathcal{P}_{\text {UNIF }}^{\delta}(n, \mathcal{L})
$$

The next lemma is essentially proved in [2]. 
Lemma 16 ( $M$-Extension Lemma). Assume that $n>n_{0}(\mathcal{L})$ and $G_{n} \in \mathcal{Q}_{\text {GOOD }}^{h}(n, \mathcal{L})$. Denote $\left(U_{1}, \ldots, U_{p}\right)$ an optimal p-partition of $V\left(G_{n}\right)$. Let $W$ be the set of vertices of $G_{n}$ having horizontal degrees at least $\varepsilon n$ in this partition. Then for every $M \in \mathcal{M}$ and every $i$ we have that $M \nsubseteq G\left[U_{i}-W\right]$.

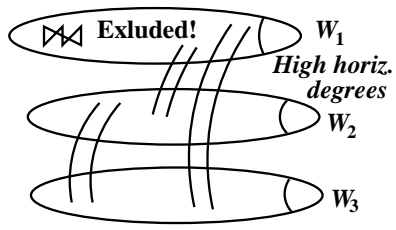

Proof. Let $W_{i}=W \cap U_{i}$. For a contradiction, assume that there is a graph $M \in \mathcal{M}$ with $M \subseteq G\left[U_{1}-W\right]$. Then there is an $L \in \mathcal{L}$ such that $L \subset\left(M \cup I_{y}\right) \otimes K_{p-1}(t, \ldots, t)$, i.e. $L$ is a 'reason' that $M \in \mathcal{M}$. So there is a vertex partition of $L$ into $L_{1}, \ldots, L_{p}$ such that $L_{1}$ spans $M$ and probably some additional isolated vertices, and each of $L_{2}, \ldots, L_{p}$ is independent in $L$. By the assumption, we can embed $M$ (spanned by $L_{1}$ ) into $U_{1}-W_{1}$. We fix such an $M$. The set $L_{1}$ is a good $(\leq t)$-tuple in $U_{1}-W_{1}$, since $v(L) \leq t$. Therefore, using that $G_{n} \in \mathcal{P}_{\text {GOOD }}^{h}(n, \mathcal{L})$ we have $\left(\Gamma^{*}\left(L_{1}\right) \cap U_{2}\right)-W_{2}$ consists of at least $\beta_{t} U_{2}-|W|$ vertices, and using that $G_{n} \in \mathcal{P}_{\mathbf{W P}}^{\vartheta}(n, \mathcal{L})$ it is at least $\beta_{t} n /(2 p)$. So an $L_{2}$ could be chosen from it. Fixing $L_{2}$, the set $L_{1} \cup L_{2}$ is a good $(\leq t)$-tuple in $\left(U_{1} \cup U_{2}\right)-W$, therefore $\Gamma^{*}\left(L_{1} \cup L_{2}\right) \cap U_{3}-W_{3}$ is 'large' and an $L_{3} \subset \Gamma^{*}\left(L_{1} \cup L_{2}\right) \cap U_{3}-W_{3}$ could be chosen. This can be continued till we find $L_{p}$ in $U_{p}$, therefore we have a copy of $L$ in $G_{n}$ as a subgraph, a contradiction.

\section{Some important lemmas}

The following easy lemma says that if the size of a subclass of the $\mathcal{L}$-free graphs can be estimated by $2^{e\left(T_{n, p}\right)-\rho n^{2}}$ then this subclass is really negligible.

Lemma 17 (Many GOOD Graphs). For any fixed $t$ and $h>0$,

$$
\left|\mathcal{Q}_{\text {GOOD }}^{h}(n, \mathcal{L})\right|>(1-o(1)) 2^{e\left(T_{n, p}\right)} \quad \text { as } \quad n \rightarrow \infty \text {. }
$$

We shall need the following simple tail estimate (see for example [1]).

Lemma 18 (Tail Estimate). If $\xi_{1}, \ldots, \xi_{m}$ are $m$ independent random 0-1 variables for which $\operatorname{Prob}\left(\xi_{i}=1\right)=u>0$, then

$$
\operatorname{Prob}\left(\sum \xi_{i}<\frac{1}{2} u m\right)<e^{-\frac{1}{2} u^{2} m}
$$

We shall use this lemma in the following setting. 
Lemma 19. Let $G_{n, 1 / 2}$ be a random graph where each edge is chosen independently, with probability $1 / 2$. Let $X:=\left\{x_{1}, \ldots, x_{r}\right\} \subseteq V\left(G_{n, 1 / 2}\right)$. Let $U \subseteq V\left(G_{n, 1 / 2}\right)$ be an m-element set disjoint from $X$. Then,

$$
\operatorname{Prob}\left(\left|\Gamma^{*}(X) \cap U\right|<\frac{m}{2^{r+1}}\right)<e^{-\beta_{r} m} .
$$

Recall that $\Gamma^{*}(X):=\cap_{x \in X} N(x)$ and $\beta_{r}=2^{-(2 r+1)}$. When we apply Lemma 19. we tend to take $|X|$ bounded, and $|U|$ linear in $n$.

Proof. Let

$$
\xi_{y}= \begin{cases}0 & \text { if } y \notin U \cap \bigcap_{i \leq r} \Gamma\left(x_{i}\right), \\ 1 & \text { if } y \in U \cap \bigcap_{i \leq r} \Gamma\left(x_{i}\right) .\end{cases}
$$

Clearly, $\left|\Gamma^{*}(X) \cap U\right|=\sum_{y} \xi_{y}$. Apply Lemma 18 with $u=2^{-r}$ :

$$
\operatorname{Prob}\left(\sum_{y} \xi_{y}<\frac{m}{2^{r+1}}\right)<e^{-0.5 \cdot 2^{-2 r} m}=e^{-\beta_{r} m} .
$$

Proof of Lemma 17. The Turán graph $T_{n, p}$ has $2^{e\left(T_{n, p}\right)}$ subgraphs. Take any of them at random: select each edge of $T_{n, p}$ independently, with probability $\frac{1}{2}$.

$\left(^{*}\right)$ We know that for all but $o\left(2^{e\left(T_{n, p}\right)}\right)$ subgraphs $G_{n} \subseteq T_{n, p}$, if $\left(U_{1}, \ldots, U_{p}\right)$ is the original partition of $T_{n, p}$, then - in the random subgraph - each $x \in U_{i}$ is joined to each $U_{j}(j \neq i)$ by at least $\frac{n}{3 p}$ edges.

(**) Similarly, if $A \subset U_{i}, B \subset U_{j}$, where $i \neq j$, and $|A|,|B|>n^{0.6}$, then in all but $o\left(2^{e\left(T_{n, p}\right)}\right)$ subgraphs $G_{n} \subseteq T_{n, p}$ has an edge between $A$ and $B$.

Restricting ourselves to these subgraphs, an optimal partition of $G_{n}$ coincides with the original partition $\left(U_{1}, \ldots, U_{p}\right)$ of $T_{n, p}$. For this partition the number of horizontal edges is 0 . If there is an other optimal partition $V_{1}, \ldots, V_{p}$, then by property $(* *)$ there is a labelling of the classes, such that $\sum_{i=1}^{p}\left|U_{i} \Delta V_{i}\right|=o(n)$. But by property $\left(^{*}\right)$ if two partitions differ then their symmetric difference is at least $n /(3 p)$, a contradiction, proving the unicity of the optimal partition. We need this because whether an $r$-tuple in $G_{n}$ is BAD or GOOD depends on the partition as well.

A standard application of Lemma 18 implies that all but $o\left(2^{e\left(T_{n, p}\right)}\right)$ subgraphs $G_{n} \subseteq T_{n, p}$ belong to $\mathcal{P}_{\mathrm{UNIF}}^{\delta}(n, \mathcal{L})$, and trivially a typical $G_{n}$ is well-partitioned, so $G_{n} \in \mathcal{P}_{\text {WP }}^{\vartheta}(n, \mathcal{L})$. Recalling that $\mathcal{Q}_{\text {GOOD }}^{h}(n, \mathcal{L})=\mathcal{P}_{\text {GOOD }}^{h}(n, \mathcal{L}) \cap \mathcal{P}_{\text {WP }}^{\vartheta}(n, \mathcal{L}) \cap$ 
$\mathcal{P}_{\text {UNIF }}^{\delta}(n, \mathcal{L})$, it remains to prove that $G_{n} \in \mathcal{P}_{\text {GOOD }}^{h}(n, \mathcal{L})$ w.h.p.. We assert that the probability that $G_{n}$ has a BAD $r$-tuple is $o(1)$ for every $r \leq t$. By Lemma 19] only $o\left(2^{e\left(T_{n, p}\right)}\right)$ subgraphs have BAD $r$-tuples. Indeed, an $r$-tuple can be chosen in at most $\left(\begin{array}{l}n \\ r\end{array}\right)$ ways; fixing this $r$-tuple $X=\left\{x_{1}, \ldots, x_{r}\right\}$, the expected size of $U_{j} \cap \Gamma^{*}(X)$ is around $(n / p) \cdot 2^{-r}$. So, for any fixed $r$-tuple $X \subset V\left(G_{n}\right)$, if $X \cap U_{j}=\emptyset$, then

$$
\operatorname{Prob}\left(\left|\Gamma^{*}(X) \cap U_{j}\right|<\frac{n}{p 2^{r+1}}\right)<e^{-n /\left(p 2^{2 r+1}\right)},
$$

and

$$
p \cdot\left(\begin{array}{l}
n \\
r
\end{array}\right) e^{-n /\left(p 2^{2 r+1}\right)}=o(1) .
$$

Definition 20 ( $\ell$-BAD graphs). For given positive integer $\ell$, let

$$
\mathcal{R}_{\mathrm{BAD}}^{\ell}(n, \mathcal{L}) \subset \mathcal{P}_{\vartheta}(n, \mathcal{L}) \cap \mathcal{P}_{\mathbf{W P}}^{\vartheta}(n, \mathcal{L}) \cap \mathcal{P}_{\mathbf{U N I F}}^{\delta}(n, \mathcal{L})
$$

be the family of graphs $G_{n}$ having an optimal partition $\left(U_{1}, \ldots, U_{p}\right)$ for which the following holds. For at least one $i \leq p$, there are pairwise disjoint BAD $(\leq t)$-tuples $X_{1}, X_{2}, \ldots, X_{s} \subseteq V\left(G_{n}\right)-U_{i}-W$, with the (same) distinguished class $U_{i}$, such that

$$
\left|\bigcup_{j \leq s} X_{j}\right| \geq \ell .
$$

The next lemma claims that, in most GOOD graphs, for a fixed optimal partition the $\mathrm{BAD}(\leq t)$-tuples can be represented by $o(n)$ vertices.

Lemma 21. For the constants fixed in Definition [6, and $\ell:=\lceil\gamma n\rceil$, there is a $\rho=\rho(\gamma)>0$ such that

$$
\left|\mathcal{R}_{\mathbf{B A D}}^{\ell}(n, \mathcal{L})\right| \leq 2^{e\left(T_{n, p}\right)-\rho n^{2}} \quad \text { for } \quad n>n_{0} .
$$

Proof. Consider a graph $G_{n} \in \mathcal{R}_{\mathrm{BAD}}^{\ell}(n, \mathcal{L})$. By definition, $G_{n}$ has an optimal partition $\left(U_{1}, \ldots, U_{p}\right)$ and a class $U_{j}$ such that there are pairwise disjoint $U_{j}$-BAD $(\leq t)$-tuples $X_{1}, \ldots, X_{s}$ with $\left|\bigcup X_{i}\right| \geq \ell$ and $s \leq \ell$. We shall use an estimate of the form

$$
\left|\mathcal{R}_{\mathbf{B A D}}^{\ell}(n, \mathcal{L})\right| \leq p \cdot p^{n} \cdot n \cdot 2^{H(\vartheta) n^{2}+1} \cdot n^{t n} \cdot \mathbb{N}_{1} \cdot \mathbb{N}_{2},
$$


where on the right-hand side of (17), $p$ stands for the number of ways of choosing a distinguished class $U_{j}, p^{n}$ is a crude upper bound on the number of (optimal) $p$-partitions, $n$ bounds the number of choices for $s$, and

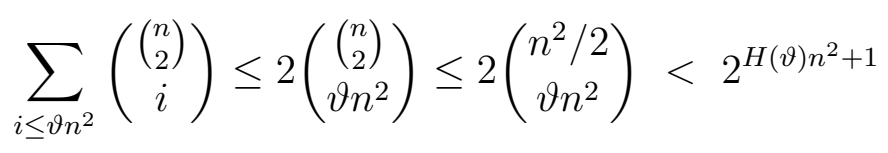

bounds the number of ways of fixing the at most $\vartheta n^{2}$ horizontal edges (as $G_{n} \in$ $\left.\mathcal{P}_{\vartheta}(n, \mathcal{L})\right)$. The explanation of the factor $n^{t n} \cdot \mathbb{N}_{1} \cdot \mathbb{N}_{2}$ is given below.

Each $X_{i}$ can be chosen in at most $\sum_{r \leq t}\left(\begin{array}{l}n \\ r\end{array}\right) \leq t\left(\begin{array}{l}n \\ t\end{array}\right) \leq n^{t}$ ways. So the system $\left\{X_{i}\right\}$ can be chosen in at most $n^{t s} \leq n^{t n}$ ways.

Let $S:=\bigcup_{i} X_{i}$. To count the graphs $G_{n} \in \mathcal{R}_{\mathbf{B A D}}^{\ell}(n, \mathcal{L})$, we fix an (optimal) partition $\left(U_{1}, \ldots, U_{p}\right)$ in each such $G_{n}$ and then the sets $X_{i}$ described above. For each $X_{i}$ put

$$
\mathbb{E}_{i}:=\bigcup_{i=1}^{s}\left\{(x, u): x \in X_{i}, u \in U_{j}\right\} \quad \text { and } E_{i}:=\left|\mathbb{E}_{i}\right|
$$

- $\mathbb{N}_{1}$ bounds the number of choices of the edges in $\mathbb{E}:=\bigcup \mathbb{E}_{i}$.

- $\mathbb{N}_{2}$ bounds the number of choices for the edges in the remaining vertical pairs, i.e. between $U_{i}$ and $U_{j}$ for $i \neq j$. If $E:=|\mathbb{E}|=\sum_{i} E_{i}$, then

$$
\mathbb{N}_{2} \leq 2^{e\left(T_{n, p}\right)-E}
$$

The key step in our proof is our bound on $\mathbb{N}_{1}$. The crude bound would be $2^{E}=$ $\prod 2^{E_{i}}$, but that is not sufficient for us. Therefore we shall sharpen this bound, checking, for each $i$, by how much we can decrease the bound $2^{E_{i}}$.

Fixing $\left(U_{1}, \ldots, U_{p}\right)$, the distinguished class $U_{j}$ and the set-pairs $\left(X_{i}, U_{j}\right)$, we count the number of ways the edges can be placed between $X_{i}$ and $U_{j}$ :

Assuming that the connection of $X_{i}$ to $U_{j}$ is random, the expected number of vertices $u \in U_{j} \cap \Gamma^{*}\left(X_{i}\right)$ (i.e. completely joined to $\left.X_{i}\right)$ is $\left|U_{j}\right| \cdot 2^{-\left|X_{i}\right|}$. However, as $U_{j}$ is bad for $X_{i}$, the number of common neighbours is below half of the expected number, therefore the number of possibilities of these connections is at least $2^{-\beta_{\left|X_{i}\right|} \cdot\left|U_{j}\right|}$ times smaller, by Lemma 19. So, taking the total number (i.e. the product of the possibilities) we have an additional factor at most

$$
\prod_{i \leq s}\left(2^{-\beta_{\left|X_{i}\right|} \cdot\left|U_{j}\right|}\right)<2^{-\sum_{i \leq s} \beta_{\left|X_{i}\right|}\left|U_{j}\right|} \leq 2^{-\beta_{t}\left|U_{j}\right|} \leq 2^{-\beta_{t} \ell n /(2 p t)}
$$


since, by (16),$s \geq \ell / t$ and as $G_{n} \in \mathcal{P}_{\mathbf{W P}}^{\vartheta}(n, \mathcal{L})$ we have $\left|U_{j}\right| \geq n /(2 p)$. Hence, using $\ell:=\lceil\gamma n\rceil$, we obtain

$$
\mathbb{N}_{1} \leq 2^{E-\beta_{t} \ell n /(2 p t)} \leq 2^{E-\gamma \beta_{t} n^{2} /(2 p t)}
$$

Combining inequalities (19), (20) and (12) with (17), we find that

$$
\left|\mathcal{R}_{\mathbf{B A D}}^{\ell}(n, \mathcal{L})\right| \leq p^{n+1} n^{t n+1} \cdot 2^{H(\vartheta) n^{2}+1+e\left(T_{n, p}\right)-E+E-\gamma \beta_{t} n^{2} /(2 p t)} \leq 2^{e\left(T_{n, p}\right)-\rho n^{2}}
$$

if $n$ is sufficiently large.

\section{Proof of Theorems [5] and 9}

Proof of Theorem 5. By Lemmas 10, 11 and 12, almost all graphs from $\mathcal{P}(n, \mathcal{L})$ are in $\mathcal{P}_{\mathbf{W P}}^{\vartheta}(n, \mathcal{L}) \cap \mathcal{P}_{\mathbf{U N I F}}^{\delta}(n, \mathcal{L})\left(\right.$ here we use that $\left.|\mathcal{P}(n, \mathcal{L})| \geq 2^{e\left(T_{n, p}\right)}\right)$. By Theorem 9. almost all graphs from $\mathcal{P}(n, \mathcal{L})$ are in $\mathcal{P}_{\text {GOOD }}^{h}(n, \mathcal{L})$, i.e. almost all of them are in $\mathcal{Q}_{\text {GOOD }}^{h}(n, \mathcal{L})$. Now Lemma 16 implies Theorem [5]

Proof of Theorem 9. The proof is based on a pseudo-symmetrization. Let

$\mathcal{P}_{\mathbf{B A D}}^{\ell}(n, \mathcal{L}):=\mathcal{P}_{\vartheta}(n, \mathcal{L}) \cap \mathcal{P}_{\mathbf{W P}}^{\vartheta}(n, \mathcal{L}) \cap \mathcal{P}_{\mathbf{U N I F}}^{\delta}(n, \mathcal{L})-\mathcal{P}_{\mathbf{G O O D}}^{h}(n, \mathcal{L})-\mathcal{R}_{\mathbf{B A D}}^{\ell}(n, \mathcal{L})$.

(Although we use $\ell=\lceil\gamma n\rceil$, we carry it in our notation.) We shall map each graph $G_{n} \in \mathcal{P}_{\mathbf{B A D}}^{\ell}(n, \mathcal{L})$ onto many $\mathcal{L}$-free graphs, changing at most $\gamma n^{2}$ edges in $G_{n}$. The set of these graphs will be denoted by $\Phi\left(G_{n}\right)$. Roughly, the main idea is that we show that for most of the graphs $H_{n}$ we have $\left|\Phi^{-1}\left(H_{n}\right)\right|=o\left(\left|\Phi\left(G_{n}\right)\right|\right)$. This will imply that $\left|\mathcal{P}_{\mathbf{B A D}}^{\ell}(n, \mathcal{L})\right|=o(|\mathcal{P}(n, \mathcal{L})|)$. We actually will show that $\mathcal{P}_{\mathbf{B A D}}^{\ell}(n, \mathcal{L})$ is an exponentially small part of $\mathcal{P}(n, \mathcal{L})$. We have to prepare the ground to carry out these ideas.

Let $G_{n} \in \mathcal{P}_{\mathbf{B A D}}^{\ell}(n, \mathcal{L})$. Since $V\left(G_{n}\right)=\{1, \ldots, n\}$ is ordered, we may define $\left(U_{1}, \ldots, U_{p}\right)$ as the "lexicographically first" optimal partition of $G_{n}$. (Of course, we do not care about the "lexicographical order": we just wish to fix one optimal partition.) As in Definition 8, let $W$ denote the set of vertices of $G_{n}$ of horizontal degree at least $\varepsilon n$ in $\left(U_{1}, \ldots, U_{p}\right)$. Let $\left\{X_{1}, \ldots, X_{s}\right\}$ be a maximal system of pairwise disjoint $\mathrm{BAD}(\leq t)$-sets toward $U_{1}$. (Again, the first one in some welldefined ordering.) We define the mapping $\Phi: \mathcal{P}_{\mathbf{B A D}}^{\ell}(n, \mathcal{L}) \mapsto 2^{[\mathcal{P}(n, \mathcal{L})]}$ as follows.

For $G_{n} \in \mathcal{P}_{\mathbf{B A D}}^{\ell}(n, \mathcal{L})$, let $\Phi\left(G_{n}\right)$ be the family of graphs obtained by joining $X=X\left(G_{n}\right):=X_{1} \cup \ldots \cup X_{s}$ to the vertices of $V\left(G_{n}\right)-U_{1}-X-W$ in any way. More precisely, let $\Phi\left(G_{n}\right)$ denote the set of graphs obtained as follows: 
First we remove all edges between $X$ and $V\left(G_{n}\right)$. Then "put" the elements of $X$ into $U_{1}$ : join the vertices of $X$ to the vertices of $V\left(G_{n}\right)-U_{1}-W-X$ arbitrarily.

To make our argument more transparent, we define $\ell$ directed graphs $\overrightarrow{\mathcal{D}}_{i}$ on the vertex set $\mathcal{P}(n, \mathcal{L})$ : in the $i^{\text {th }}$ graph $\overrightarrow{\mathcal{D}}_{i}$, there is an edge from $G_{n}$ to $H_{n}$, if $G_{n} \in \mathcal{P}_{\mathbf{B A D}}^{\ell}(n, \mathcal{L}), H_{n} \in \Phi\left(G_{n}\right)$, and $\left|X\left(G_{n}\right)\right|=i$. Then our aim is to show that in each $\overrightarrow{\mathcal{D}}_{i}$ the outdegrees are large and the indegrees are small.

Perhaps the most important property of this map is that $\Phi\left(G_{n}\right) \subset \mathcal{P}(n, \mathcal{L})$ : the graphs in $\Phi\left(G_{n}\right)$ are $\mathcal{L}$-free. Note that this is the part of the proof where we could not avoid using that $\mathcal{L}$ is finite.

To show that any $H_{n} \in \Phi\left(G_{n}\right)$ is $\mathcal{L}$-free, observe that if we obtained some $L \in \mathcal{L}$ during our "symmetrization", i.e. if $L \subset H_{n}$, then the original $G_{n}$ also contained an $L^{\prime} \simeq L$. Indeed, $V(L)$ in $H_{n}$ can be partitioned into four parts:

(i) $R_{*}=V(L) \cap X \neq \emptyset$,

(ii) $C_{*}=V(L) \cap U_{1}-X$ : the remaining part of $L$ in $U_{1}$,

(iii) $W_{*}=V(L) \cap W-U_{1}-X$,

(iv) $L_{*}=V(L)-U_{1}-W-X$.

$\boldsymbol{R}_{*} \quad$ Observe that $L_{*}$ was a GOOD $(\leq t)$-tuple in $G_{n}$,

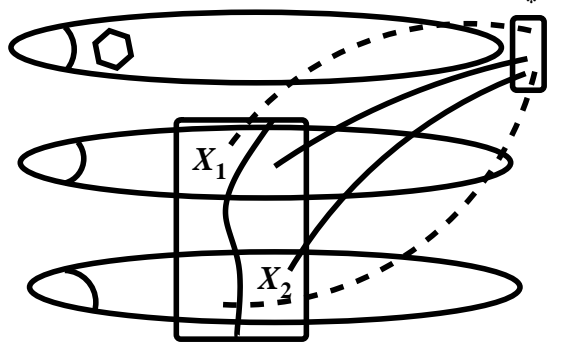
otherwise $\left\{X_{1}, \ldots, X_{s}\right\}$ was not maximal. Hence $\left|\Gamma^{*}\left(L_{*}\right) \cap U_{1}-X-W\right|>|V(L)|$. Therefore we can fix a set $Y \subset \Gamma^{*}\left(L_{*}\right) \cap U_{1}-X-W$ with $|Y|=\left|R_{*}\right|$. In $H_{n}$ there is no edge between $X$ and $W$, and between $X$ and $U_{1}$. So in $G_{n}$ the graph spanned by $C_{*} \cup W_{*} \cup L_{*} \cup Y$ contains an $L$. This contradiction shows that $L \nsubseteq H_{n}$.

The next step is to give a lower bound on the outdegrees in $\overrightarrow{\mathcal{D}}_{i}$, i.e. to estimate $\left|\Phi\left(G_{n}\right)\right|$, given that $\left|X\left(G_{n}\right)\right|=i$. Creating the graphs in $\Phi\left(G_{n}\right)$, for any pair $(a, b)$ with $a \in X$ and $b \in V\left(G_{n}\right)-U_{1}-X-W$, we may include or exclude $(a, b)$ as an edge. Hence, using that $|X| \leq \gamma n \leq \ell$ and that by $G_{n} \in \mathcal{P}_{\mathbf{W P}}^{\vartheta}(n, \mathcal{L})$ the classes are not big, i.e. $\left|U_{1}\right| \leq(1 / p+\sqrt{\vartheta} \log (1 / \vartheta)) n$, we have

$$
\begin{aligned}
\left|\Phi\left(G_{n}\right)\right| & =2^{|X| \cdot\left(n-\left|U_{1}\right|-|X|-|W|\right)} \geq 2^{i n(1-1 / p-\sqrt{\vartheta} \log (1 / \vartheta)-i / n-o(1))} \\
& \geq 2^{i n(1-1 / p-\gamma-\sqrt{\vartheta} \log (1 / \vartheta)-o(1))} .
\end{aligned}
$$

Our final aim is to bound the indegrees in $\overrightarrow{\mathcal{D}}_{i}$. In order to do this, first we bound the number of optimal partitions of graphs in $\mathcal{P}(n, \mathcal{L})$. Note that during the operation $\Phi$ the number of horizontal edges in the optimal partitions does not increase, hence $G_{n} \in \mathcal{P}_{\vartheta}(n, \mathcal{L})$ implies $\Phi\left(G_{n}\right) \subset \mathcal{P}_{\vartheta}(n, \mathcal{L})$. 
Lemma 22. Given a graph $H_{n} \in \mathcal{P}_{\vartheta}(n, \mathcal{L})$, the number of optimal partitions of the graphs in $\Phi^{-1}\left(H_{n}\right)$ is at most $2^{2 p H(\delta p+2 \gamma p) n}$, where the same partition obtained from different graphs are counted only once.

Proof. Let $H_{n} \in \mathcal{P}_{\vartheta}(n, \mathcal{L})$ and $G_{1}, G_{2} \in \Phi^{-1}\left(H_{n}\right)$, where $G_{1}=G_{2}$ is allowed. Recall that the domain of $\Phi$ was a subset of $\mathcal{P}_{\mathbf{U N I F}}^{\delta}(n, \mathcal{L}) \cap \mathcal{P}_{\mathbf{W P}}^{\vartheta}(n, \mathcal{L})$. Let $\left(U_{1}, \ldots, U_{p}\right)$ be an optimal partition of $G_{1}$ and $\left(V_{1}, \ldots, V_{p}\right)$ of $G_{2}$. For $j=1,2$, let $X_{j}$ be the $i$-set of the vertices of $G_{j}$ incident with the edges that were changed by $\Phi$ to obtain $H_{n}$. Note that as the optimal partitions of $G_{i}$ are $\delta$-lower regular and balanced, for every $a$ there is at most one $b$ such that $\left|V_{a} \cap U_{b}\right|>\delta n+2 i$ (for $1 \leq a, b \leq p$ ). Otherwise, if say $\left|V_{a} \cap U_{b_{1}}\right|,\left|V_{a} \cap U_{b_{2}}\right|>\delta n+2 i$, then $e_{G_{2}}\left(V_{a} \cap U_{b_{1}}, V_{a} \cap U_{b_{2}}\right) \geq \delta^{2} n^{2} \geq 4 \vartheta n^{2}$, contradicting $G_{2} \in \mathcal{P}_{\vartheta}(n, \mathcal{L})$.

This implies the existence of a labelling of the classes such that for every $a$, $1 \leq a \leq p$ we have $\left|V_{a}-U_{a}\right| \leq(p-1)(\delta n+2 i)$.

As for a given optimal partition $\left(U_{1}, \ldots, U_{p}\right)$ and $D_{a}:=V_{a}-U_{a}$, the partition $\left(V_{1}, \ldots, V_{p}\right)$ is determined, the number of optimal partitions $\left(V_{1}, \ldots, V_{p}\right)$ is bounded by the number of ways the difference sets $D_{a}$ can be chosen:

$$
\left(\sum_{j=0}^{(p-1)(\delta n+2 i)}\left(\begin{array}{c}
n \\
j
\end{array}\right)\right)^{p}<\left(\begin{array}{c}
n \\
\delta p n+2 i p
\end{array}\right)^{p}<2^{2 p H(\delta p+2 p \gamma) n} .
$$

Our next step to bound the indegrees in $\overrightarrow{\mathcal{D}}_{i}$ is to give an upper bound on the number of ways of choosing the BAD $(\leq t)$-tuples. The number of ways of choosing the index $j$ in $U_{j}$ which is the distinguished class is bounded by $p$. Then we can fix $s$, the number of sets in $\left\{X_{1}, \ldots, X_{s}\right\}$ in less than $n$ ways. The number of ways to choose $\left\{X_{1}, \ldots, X_{s}\right\}$ is less than $n^{t s}$. Then for each $x \in \bigcup X_{\ell}$ we may choose the class $U_{m}$ containing $x$ in $p$ ways: altogether in $p^{i}$ ways. By Lemma 22, the number of ways to fix an optimal partition of $G_{n} \in \Phi^{-1}\left(H_{n}\right)$ is at most $2^{2 p H(p \delta+2 p \gamma) n}$.

Fixing an optimal partition of $G_{n}$ and the sets $X_{1}, \ldots, X_{s}$, we know all edges of $G_{n}$, except the ones adjacent to $X$. Note that by the definition of a BAD-tuple, each $x \in X$ had horizontal degree at most $\varepsilon n$. Thus the number of ways of adding the horizontal edges with at least one end point in $X$ is at most

$$
\left(\sum_{j=0}^{\varepsilon n}\left(\begin{array}{c}
n \\
j
\end{array}\right)\right)^{i} \leq 2^{i} \cdot\left(\begin{array}{c}
n \\
\varepsilon n
\end{array}\right)^{i} \leq 2^{i+H(\varepsilon) i n}
$$


We shall use that, as $G_{n} \in \mathcal{P}_{\mathbf{W P}}^{\vartheta}(n, \mathcal{L})$, we have

$$
\frac{n}{p}-\sqrt{\vartheta} \log (1 / \vartheta) n \leq u_{\min }:=\min _{1 \leq j \leq p}\left\{\left|U_{j}\right|\right\} \leq u_{\max }:=\max _{1 \leq j \leq p}\left\{\left|U_{j}\right|\right\} \leq \frac{n}{p}+\sqrt{\vartheta} \log (1 / \vartheta) n .
$$

For a vertex $x \in X \cap U_{j}$ the number of possibilities of having the edge set in $G_{n}$ between $x$ and $V\left(G_{n}\right)-U_{1}-U_{j}$ is at most $2^{n-2 u_{m i n}}$. So the total number of ways of joining the elements of $X$ to the rest of the graph excluding to its own class and $U_{1}$ is at most

$$
2^{|X|\left(n-2 u_{\min }\right)} .
$$

For any $j \leq s$ as $\left|\Gamma^{*}\left(X_{j}\right) \cap U_{1}\right|$ is smaller than half of its expected value in a random graph, by Chernoff's inequality (Lemma 19), the number of ways having the edges between $X_{j}$ and $U_{1}$ is at most

$$
2^{u_{\max }\left(\left|X_{j}\right|-\beta_{t}\right)}
$$

We have to consider this for each $X_{j}$. Note that $\lceil i / t\rceil \leq s \leq i$. Putting these together, we have the following upper bound on the maximum indegree in $\overrightarrow{\mathcal{D}}_{i}$ :

$$
\begin{aligned}
2^{2 p H(p \delta+2 \gamma p) n} & \cdot p \cdot n \cdot n^{t s} \cdot p^{i} \cdot 2^{i+H(\varepsilon) i n} \cdot 2^{|X|\left(n-2 u_{\min }\right)} \cdot \prod_{j=1}^{s} 2^{u_{\max }\left(\left|X_{j}\right|-\beta_{t}\right)} \\
\leq & 2^{i n\left[2 p H(p \delta+2 p \gamma)+o(1)+H(\varepsilon)+1-2 / p+2 \sqrt{\vartheta} \log (1 / \vartheta)+1 / p+\sqrt{\vartheta} \log (1 / \vartheta)-s \beta_{t} /(i p)\right]} \\
\leq & 2^{i n\left[1-1 / p+o(1)+2 p H(p(\delta+2 \gamma))+H(\varepsilon)+3 \sqrt{\vartheta} \log (1 / \vartheta)-\beta_{t} /(p t)\right]} .
\end{aligned}
$$

With this bound our proof is essentially complete. Recall that the outdegree was bounded from below by

$$
2^{i n(1-1 / p-\gamma-\sqrt{\vartheta} \log (1 / \vartheta)-o(1))}
$$

Comparing the upper bound on the indegree and (22), the outdegree estimate in $\overrightarrow{\mathcal{D}}_{i}$, and using (13), we see that the ratio of them is at least $2^{\beta_{t} n /(2 p t)}$, i.e. the number of BAD graphs with $|X|=i$ is at most $\left|\mathcal{P}_{\vartheta}(n, \mathcal{L})\right| \cdot 2^{-\beta_{t} n /(2 p t)}$. Since $i \leq n$, the number of BAD graphs is at most, $\left|\mathcal{P}_{\vartheta}(n, \mathcal{L})\right| \cdot 2^{-\beta_{t} n /(3 p t)}$, say. Considering only the "good graphs" we neglected fewer than $4 \cdot 2^{e\left(T_{n, p}\right)-\rho n^{2}}$ (other) graphs. This completes the proof.

\section{Acknowledgement}

We are indebted to the referees for their careful reading and numerous suggestions that improved the presentation of the paper. 
Balogh-Bollobás-Simonovits: Typical structure, September 4, 2008

\section{References}

[1] N. Alon and J.H. Spencer, The Probabilistic Method, 2nd ed., John Wiley and Sons, Inc., New York, 2000.

[2] J. Balogh, B. Bollobás, and M. Simonovits, The number of graphs without forbidden subgraphs, J. Combin. Theory Ser. B 91 (2004), 1-24.

[3] B. Bollobás, Extremal Graph Theory, Academic Press, London, (1978).

[4] B. Bollobás, Extremal Graph Theory, Handbook of Combinatorics, eds Graham, Groetschel, Lovász MIT Press Press, (1996) pp. 1231-1292.

[5] P. Erdős, On some new inequalities concerning extremal properties or graphs, Theory of Graphs (edited P. Erdős and G. Katona), Academic Press, New York, 1968, pp. 77-81.

[6] P. Erdős, P. Frankl, and V. Rödl, The asymptotic number of graphs not containing a fixed subgraph and a problem for hypergraphs having no exponent, Graphs and Combinatorics, 2 (1986), 113-121.

[7] Z. Füredi, Turán type problems, in Surveys in Combinatorics (1991), Proc. of the 13th British Combinatorial Conference, (ed. A. D. Keedwell) Cambridge Univ. Press. London Math. Soc. Lecture Note Series 166 (1991), 253-300.

[8] M. Simonovits, A method for solving extremal problems in graph theory, stability problems, Theory of Graphs (Proc. Colloq. Tihany, 1966), (edited by P. Erdős and G. Katona), Academic Press, New York, and Akad. Kiadó, Budapest, 1968, 279-319.

[9] M. Simonovits, Extremal Graph Theory, Selected Topics in Graph Theory, (ed. by Beineke and Wilson) Academic Press, London, New York, San Francisco, (1983), 161-200.

[10] M. Simonovits, Paul Erdös' influence on extremal graph theory, The mathematics of Paul Erdős, II, 148-192, Algorithms Combin., 14, Springer, Berlin, 1997.

[11] E. Szemerédi, Regular partitions of graphs, Colloques Internationaux C. N. R. S. 260, in Problèmes combinatoires et théorie des graphes. Proc. Colloq. Orsay, Éditions C. N. R. S; Paris, (1978), 399-401. 